\title{
On Possibilistic Multi-Objective Multi-item Solid Transportation Problems
}

\author{
H. A. Khalifa \\ Department of Operations Research \\ Institute of Statistical Studies and Research \\ Cairo University, Cairo, Egypt
}

\begin{abstract}
The solid transportation problem (STP) arises when bounds are given on three item properties. These properties are usually: sources destination and type of product or mode of transport.

In this paper, a possibilistic multi-objective multi-item solid transportation problem (Poss MOMISTP) is studied. The problem is considered by incorporating possibilistic data into the objective functions coefficients. The efficient solutions and the stability of Poss MOMISTP problem are investigated. The concept of $\alpha$-Possibly efficient is introduced in which the ordinary efficient solution is $\alpha$-tended based on the $\alpha$ level of possibilistic variables. A necessary and sufficient condition for such solution is established. A relationship between solutions of possibilistic levels is constructed. The stability set of the first kind corresponding to one solution of the $\alpha$ - level of possibilistic variables is determined. An illustrative numerical example is given in the sake of the paper to clarify the obtained results.
\end{abstract}

\section{Keywords}

Multi-objective multi-item solid transportation problems; Possibilistic variables; $\alpha$-possibly efficient; Parametric analysis

\section{INTRODUCTION}

The solid transportation problem (STP) is a generalization of the wall-known transportation problem (TP) in which three item properties are taken into account in the constraint set instead of two (source and destination). The STP was first introduced by Shell (1955). Haley (1962) showed a comparison of the STP and the classical TP and then applied modified method to solve the STP. Jimenez and Verdegay (1998) present two types of uncertain STP in which the considered data are interval numbers and fuzzy numbers, respectively.

Hussein (1998) studied the complete set of $\alpha$-possibly efficient solutions of multiple objective transportation problems with possibilistic objectives functions coefficients. Ammar and Youness (2005) introduced the solutions of multiobjective TP problem with fuzzy objective, fuzzy sources, and fuzzy destinations. Pandion and Anuradha(2010) propose a new approach for solving STP. Ojha et al. (2010) introduced a TP with fixed charges and vehicle costs where all unit discount (AUD) incremental quantity discount (IQD) or combinations of AUD and IQD on the price depending upon the amount is offered and varies on the choice of origin, destination and conveyance and solved the problem using Genetic Algorithm. Kundu et al. (2013) modeled a multiobjective multi-item solid transportation problem with fuzzy coefficient of the objectives and constraints and then solved by two different methods. Ammar and Khalifa (2014) introduced the multiobjective solid transportation problem with fuzzy parameters. Ammar and Khalifa (2015) Studied the multiobjective solid transportation problems with possibilistic parameters.

In this paper, multi-objective multi-item solid transportation problem with possibilistic objective functions coefficients is studied. The concept of $\alpha$-possibly efficient and $\alpha$ parametric efficient solutions are introduced, and the relation between the two previous solution is given. A parametric analysis is used to characterize the set of all $\alpha$-parametric efficient solution. A solution procedure to determine the stability set of the first kind corresponding to one parametric efficient solution of Poss MOMISTP is presented. A numerical example is given for studying the Poss-MOMISTP, we present some definitions and some notions related to the topic.

\section{PRELIMINARIES}

Definition 1. A possibilistic variable $y$ on $U$ is a variable characterized by a possibility distribution $\pi_{y}(U)$. This means that, if $y$ is a variable having values in $U$, then a possibility distribution $\pi_{y}$ associated with $y$ may be viewed as a fuzzy constraint on the values that may be assigned to $y$. Such a distribution is characterized by a possibility distribution function:

$\pi_{y}: U \rightarrow[0,1]$ which associated with each $u \in U$ the degree of compatibility of the variable $y$ with the realization $u \in U$.

If $U$ is a Cartesian product of $U_{1}, \ldots, U_{n}$, then $\pi_{y}\left(u_{1}, \ldots, u_{n}\right)$ is an $n$-ary possibility distribution, i.e., $\pi_{y}(u) \equiv\left(\pi_{y_{1}}\left(u_{1}\right), \ldots, \pi_{y_{n}}\left(u_{n}\right)\right)$.

Definition 2. The $\alpha$-cut of a possibilistic variable $y$ is defined as:

$$
y_{\alpha}=\left\{u \in U: \pi_{y}(u) \geq \alpha, \alpha \in[0,1]\right\} .
$$

Definition 3. A possibility distribution $\pi_{y}$ on $U$ is said to be convex if: 
$\pi_{y}\left(\gamma u^{1}+(1-\gamma) u^{1}\right) \geq \min \left(\pi_{y}\left(u^{1}\right), \pi_{y}\left(u^{2}\right)\right), \forall u^{1}, u^{2} \in U$, $\gamma \in[0,1]$.

Definition 4. The support of a possibilistic variable $y$ is defined as:

$\operatorname{supp}(y)=\left\{u \in U: \sup g p_{y}(u)>0 ; \forall \varepsilon>0\right\}, u \in N_{\varepsilon}(u)$,

where $N_{\varepsilon}(u)=\{u \in U:\|u-U\|<\varepsilon\}$.

Lemma 1. The $\operatorname{supp}(y)$ is a closed set on $U$.

\section{PROBLEM FORMULATION}

Consider the following possibilistic multi-objective multiitem solid transportation problems (Poss MOMISTP)

(Poss MOMISTP) $\min z_{r}\left(x, \tilde{c}^{r}\right)=\tilde{c}^{r} x$

$=\sum_{p=1}^{t} \sum_{i=1}^{m} \sum_{j=1}^{n} \sum_{k=1}^{\ell} \tilde{c}_{i j k}^{r p} \underset{i j k}{p}, r=1, \ldots, q$

subject to

$x \in G=\left\{x \in R^{q(t * m * n * \ell)}: \sum_{j=1}^{n} \sum_{k=1}^{\ell} x_{i j k}^{p} \leq a_{i}^{p}, i=1, \ldots, m ; p=1, \ldots, t\right.$,

$\sum_{i=1}^{m} \sum_{k=1}^{\ell} x_{i j k}^{p} \geq b_{j}^{p}, j=1, \ldots, n ; p=1, \ldots, t ; \sum_{p=1}^{t} \sum_{i=1}^{n} \sum_{j=1}^{n} x_{i j k}^{p} \leq e_{k}$,

$k=1, \ldots, \ell ; \sum_{i=1}^{m} a_{i}^{p} \geq \sum_{j=1}^{n} b_{j}^{p}, p=1, \ldots, t ; \sum_{k=1}^{\ell} e_{k} \geq \sum_{p=1}^{t} \sum_{j=1}^{n} b_{j}^{p}$,

and $\left.x_{i j k}^{p} \geq 0, p=1, \ldots, t, i=1, \ldots, m ; j=1, \ldots, n ; k=1, \ldots, \ell\right\}$

Where, $p(=1, \ldots, t)$ items are to be transported from $m$ origins $O_{i}(i=1, \ldots, m)$ to $n \quad$ destinations $D_{i}(i=1, \ldots, m)$ by means of $k(=1, \ldots, \ell)$ different modes of transportation (conveyance). For the objective $z_{r}, \tilde{c}_{i j k}^{r p}$ represents possibilistic unit transportation penalty on $R$ from $i^{\text {th }}$ origin to $j^{\text {th }}$ destination by $k^{\text {th }}$ conveyance from $p^{\text {th }}$ item which is an uncertain quantity can be characterized by possibility distributions $\pi_{\tilde{c}_{i j k}^{r p}}(r=1, \ldots, q ; p=1, \ldots, t ; i=1, \ldots, m ; j=1, \ldots, n ; k=1, \ldots, \ell)$ , $a_{i}^{p}$ and $b_{j}^{p}$ represent total supply of $i^{\text {th }}$ origin and total demand of $j^{\text {th }}$ destination, respectively for $p^{\text {th }}$ item, and $e_{k}$ is the total capacity of $k^{\text {th }}$ conveyance. It is assumed that all possibility distributions involved in Poss MOMISTP are

Definition 5. ( $\alpha$-possibly efficient solution). $\mathrm{A}$ point $x^{*} \in G$ is said to be $\alpha$-possibly efficient solution for Poss MOMISTP if there is no point $x \in G$ such that:
$\operatorname{Poss}\left(z_{1}\left(x, \tilde{c}^{1}\right) \leq z_{1}\left(x^{*}, \tilde{c}^{1}\right), \ldots, z_{r-1}\left(x, \tilde{c}^{(r-1)}\right) \leq z_{r-1}\left(x^{*}, \tilde{c}^{(r-1)}\right)\right.$, $z_{r}\left(x, \tilde{c}^{r}\right)<z_{r}\left(x^{*}, \tilde{c}^{r}\right), z_{r+1}\left(x, \tilde{c}^{(r+1)}\right) \leq z_{r+1}\left(x^{*}, \tilde{c}^{(r+1)}\right), \ldots$,

$\left.z_{q}\left(x, \tilde{c}^{q}\right) \leq z_{q}\left(x, \tilde{c}^{q}\right)\right) \geq \alpha$

where Poss denotes possibility.

On account of the extension principle,

Poss $\left(z_{1}\left(x, \tilde{c}^{1}\right) \leq z_{1}\left(x^{*}, \tilde{c}^{1}\right), \ldots, z_{r-1}\left(x, \tilde{c}^{(r-1)}\right) \leq z_{r-1}\left(x^{*}, \tilde{c}^{(r-1)}\right)\right.$, $z_{r}\left(x, \tilde{c}^{r}\right)<z_{r}\left(x^{*}, \tilde{c}^{r}\right), z_{r+1}\left(x, \tilde{c}^{(r+1)}\right) \leq z_{r+1}\left(x^{*}, \tilde{c}^{(r+1)}\right), \ldots$, $\left.z_{q}\left(x, \tilde{c}^{q}\right) \leq z_{q}\left(x, \tilde{c}^{q}\right)\right)=\sup _{\left(c^{1}, \ldots, c^{q}\right) \in C} \min \left(\pi_{\tilde{c}^{1}}\left(c^{1}\right), \ldots, \pi_{\tilde{c}^{r-1}}\left(c^{r-1}\right)\right.$,

$\pi_{\tilde{c}^{r}}\left(c^{r}\right), \pi_{\tilde{c}^{r+1}}\left(c^{r+1}\right), \ldots, \pi_{\tilde{c}^{q}}\left(c^{q}\right)$,

where

$C=\left\{\left(c^{1}, \ldots, c^{q}\right) \in R^{q(t * m * n * \ell)}: z_{1}\left(x, c^{1}\right) \leq z_{1}\left(x^{*}, c^{1}\right), \ldots\right.$, $z_{r-1}\left(x, c^{r-1}\right) \leq z_{r-1}\left(x^{*}, c^{r 1}\right), z_{r}\left(x, c^{r}\right) \leq z_{r}\left(x^{*}, c^{r}\right), z_{r+1}\left(x, c^{r+1}\right) \leq$

$\left.z_{r+1}\left(x^{*}, c^{r+1}\right), \ldots, z\left(x, c^{q}\right) \leq z_{q}\left(x^{*}, c^{q}\right)\right\}$

and

$\pi_{\tilde{c}^{r}}\left(c^{r}\right),(r=1, \ldots, q)$

are $(t * m * n * \ell)$-ary possibility distributions.

\section{CHARACTERIZING OF $\beta$-Possibility EFFICIENT SOLUTIONS FOR POSS MOMISTP}

For characterizing the $\beta$-Possibility efficient solution for Poss MOMISTP let us consider the following $\beta$-parametric multi-objective multi-item solid transportation problems ( $\beta$ PMOMISTP).

$\left(\beta\right.$-PMOMISTP) $\min z_{r}\left(x, c^{r}\right)=c^{r} x$

$=\sum_{p=1}^{t} \sum_{i=1}^{m} \sum_{j=1}^{n} \sum_{k=1}^{\ell} c_{i j k}^{r p} x_{i j k}^{p}, r=1, \ldots, q$

subject to

$x \in G$, and $c_{i j k}^{r p} \in\left(\tilde{c}_{i j k}^{r p}\right)_{\beta}, \beta \in[0,1]$, where $\left(\tilde{c}_{i j k}^{r p}\right)_{\beta}$ denotes the $\beta$-cut of the possibilistic variable $c_{i j k}^{r p}$. By the convexity assumption $\pi_{\tilde{c}_{i j k}^{r p}}$ and, $\left(\tilde{c}_{i j k}^{r p}\right)_{\beta},(r=1, \ldots, q ; p=1, \ldots, t ; i=1, \ldots, m ; j=1, \ldots, n$; $k=1, \ldots, \ell)$ are intervals that will be denoted as 
$\left[\left(c_{i j k}^{r p}(\beta)\right)^{L},\left(c_{i j k}^{r p}(\beta)^{U}\right]\right.$. Let $\phi_{\beta}^{r}$ be the set of $t * m * n * \ell \quad$ matrices $\quad c^{r}=\left(c_{i j k}^{r p}\right)_{\beta} \quad$ with $c_{i j k}^{r p} \in\left[\left(c_{i j k}^{r p}(\beta)^{L}, c_{i j k}^{r p}(\beta)^{U}\right], \quad r=1, \ldots, q\right.$; $p=1, \ldots, t ; i=1, \ldots, m ; j=1, \ldots, n ; k=1, \ldots, \ell$ . It is clear that $\alpha$-PMOMISTP may be rewritten in the following form:

$P(\beta): \min z_{r}\left(x, c^{r}\right), r=1, \ldots, q$

subject to

$x \in G$ and $c^{r} \in \phi_{\beta}^{r}, r=1, \ldots, q$.

Definition 6. $\beta$-parametric efficient solution: A point $x^{*} \in G$ is said to be an $\beta$-parametric efficient solution for problem $\left(P(\beta) P(\alpha)_{\beta}\right)$ if and only if there are no $x \in G \quad$ and $\quad c_{i j k}^{r p} \in \phi_{\beta}^{r} \quad$ such that $z_{r}\left(x, c^{r}\right) \leq z_{r}\left(x^{*}, c^{r}\right)$, for all $r=1, \ldots, q$ and strict inequality holds for at least one $r$.

Theorem 1. A point $x^{*} \in G$ is an $\beta$-possibly efficient solution for problem Poss MOMISTP if and only if $x^{*} \in G$ is an $\beta$-parametric efficient solution for problem $P(\beta)$.

Proof. Necessity. Let $x^{*} \in G$ be an $\alpha$-possibly efficient solution for problem Poss MOMIST and $x^{*} \in G(\alpha)$ be not an $\beta$-parametric efficient solution for problem $P(\beta)$.

Then there are $x^{1} \in G$ and $t^{r} \in \phi_{\alpha}^{r}, r=1, \ldots, q$ such that:

$z_{d}\left(x^{1}, t^{d}\right) \leq z_{d}\left(x^{*}, t^{d}\right)$,

for all $d \in\{1, \ldots, q\}, r \in\{1, \ldots, q\}$,

such that

$$
z_{r}\left(x^{1}, t^{r}\right)<z_{r}\left(x^{*}, t^{r}\right) .
$$

As $t^{r} \in \phi_{\alpha}^{r}, r=1, \ldots, q$, we have

$$
\text { Poss }\left(z_{1}\left(x^{1}, \tilde{c}^{1}\right) \leq z_{1}\left(x^{*}, \tilde{c}^{1}\right), \ldots, z_{r-1}\left(x^{1}, \tilde{c}^{(r-1)}\right) \leq z_{r-1}\left(x^{*}, \tilde{c}^{(r-1)}\right),\right.
$$$$
z_{r}\left(x^{1}, \tilde{c}^{r}\right)<z_{r}\left(x^{*}, \tilde{c}^{r}\right), z_{r+1}\left(x^{1}, \tilde{c}^{(r+1)}\right) \leq z_{r+1}\left(x^{*}, \tilde{c}^{(r+1)}\right), \ldots,
$$$$
\left.z_{q}\left(x^{1}, \tilde{c}^{q}\right) \leq z_{q}\left(x^{*}, \tilde{c}^{q}\right)\right) \geq \alpha \text {. }
$$

This contradicts that $x^{*} \in G(\alpha)$ is an $\beta$-possibly efficient solution for problem Poss MOMISTP.
Sufficiency: Let $x^{*} \in G$ be an $\beta$-parametric efficient solution for problem $(P(\beta))$ and $x^{*} \in G$ be not an $\beta$ -possibly efficient solution for problem Poss MOMISTP. Then there are $x^{2} \in G$ and $r=\{1, \ldots, q\}$ such that:

$$
\begin{aligned}
& \text { Poss }\left(z_{1}\left(x^{2}, \tilde{c}^{1}\right) \leq z_{1}\left(x^{*}, \tilde{c}^{1}\right), \ldots, z_{r-1}\left(x^{2}, \tilde{c}^{(r-1)}\right) \leq z_{r-1}\left(x^{*}, \tilde{c}^{(r-1)}\right),\right. \\
& z_{r}\left(x^{2}, \tilde{c}^{r}\right)<z_{r}\left(x^{*}, \tilde{c}^{r}\right), z_{r+1}\left(x^{2}, \tilde{c}^{(r+1)}\right)<z_{r+1}\left(x^{*}, \tilde{c}^{(r+1)}\right), \ldots, \\
& z_{q}\left(x^{2}, \tilde{c}^{q}\right)<z_{q}\left(x^{*}, \tilde{c}^{q}\right) \geq \beta
\end{aligned}
$$

i.e.,

$$
\sup _{\left(c^{1}, \ldots, c^{q}\right) \in \bar{C}} \min \left(\pi_{\tilde{c}^{1}}\left(c^{1}\right), \ldots,\left(\pi_{\tilde{c}^{1}}\left(c^{1}\right)\right) \geq \beta\right.
$$

Where,

$\bar{C}=\left\{\left(c^{1}, \ldots, c^{q}\right) \in R^{(t * m * n * \ell)}: z_{r}\left({ }^{2}, c^{1}\right) \leq z_{1}\left(x^{*}, c^{1}\right), \ldots, z_{r-1}\left(x^{2}, c^{r-1}\right) \leq\right.$ $\left.z_{r-1}\left(x^{*}, c^{r-1}\right), z_{r}\left(x^{2}, c^{r}\right)<z_{r}\left(x^{*}, c^{r}\right), \ldots, z_{q}\left(x^{2}, c^{q}\right) \leq z_{q}\left(x^{*}, c^{q}\right)\right\}$.

For the supremum in (4) to exist, there is $\left(e^{1}, \ldots, e^{q}\right) \in \bar{C}$ with

$$
\min \left(\pi_{\tilde{c}^{1}}\left(e^{1}\right), \ldots, \pi_{\tilde{c}^{q}}\left(e^{q}\right)\right)<\beta,
$$

then,

$$
\sup _{\left(e^{1}, \ldots, e^{q}\right) \in \bar{C}} \min \left(\pi_{\tilde{c}^{1}}\left(e^{1}\right), \ldots, \pi_{\tilde{c}^{q}}\left(e^{q}\right)\right)<\beta .
$$

This contradicts (4). Then there is $\left(e^{1}, \ldots, e^{q}\right) \in \bar{C}$, satisfying

$$
\min \left(\pi_{\tilde{c}^{1}}\left(e^{1}\right), \ldots, \pi_{\tilde{c}^{q}}\left(e^{q}\right)\right) \geq \beta
$$

i.e.,

$$
e^{q} \in \phi_{\beta}^{r}, \quad r=1, \ldots, q
$$

(4) and (6) leads to the contradiction of the efficiency of $x^{*} \in G$ for problem $\left(P_{\beta}\right)$.

Let $E_{\beta}(Y)$ be the set of all possibly efficient solutions for problem Poss MOMISTP and $\quad \operatorname{Eff}\left(\phi_{\beta}\right)$ be the set of all $\beta$-parametric efficient solutions for problem $(\alpha P)$ at contain $\beta \in[0,1]$.

Theorem 2. (a) $E_{\beta_{1}}(Y) \subseteq E_{\beta_{2}}(Y)$ if and only if $\beta_{1} \supseteq \beta_{2}$,

(b) $E_{0}(Y)=\bigcup_{\beta \in[0,1]} E_{\beta}(Y)$. 
Problem $P(\beta)$ will be treated using the weighting approach, i.e., by defining the following problem:

$P(\beta, w): \min \sum_{r=1}^{q} w_{r} z_{r}\left(x, c^{r}\right)=\sum_{r=1}^{q} w_{r} c^{r} x$ subject to

$x \in G, c^{r} \in \phi_{\beta}^{r}, \quad r=1, \ldots, q$, and

$w \in W=\left\{w \in R: \sum_{r=1}^{q} w_{r}=1, w_{r} \geq 0\right\}$.

We see that $x^{*}$ is an $\beta$-parametric efficient solution of problem $(P(\beta))$ if there exists $w^{*} \in W$ such that $x^{*}$ is the unique $\beta$-possibly optimal solution of problem $P\left(\beta, w^{*}\right)$.

\section{PARAMETRIC ANALYSIS}

For characterizing the set of all 0-possibly efficient solution to Poss MOMISTP $\operatorname{problem}\left(E_{\beta}(Y)\right)$, we use the decomposition of the parametric space. For the sake of parametric analysis. $P(0, w)$ problem can be written in the following form (Bazaraa et al., (1990) and Steuer (1986)):

$P(0, w): \min \sum_{r=1}^{q} w_{r}\left(\left(c^{r L}(0)+\theta c^{r U}(0) x\right.\right.$

subject to

$A x=b, \quad x \geq 0$,

$w \in W \quad$ and $\quad \theta \in[0,1]$.

Decomposing $A$ into $[B, N B], c^{r L}(0) \quad$ into $\left[c_{B}^{r L}(0), c_{N B}^{r L}(0)\right]$ and $\left[c_{B}^{r U}(0), c_{N B}^{r U}(0)\right]$, we get

$B x_{B}+N B x_{N B}=b$,

$z-\sum_{r=1}^{q} w_{r}\left[\left(c_{B}^{r L}(0)+\theta c_{B}^{r U}(0)\right) x_{B}+\left(c_{N B}^{r L}(0)+\theta c_{N B}^{r U}(0)\right) x_{B}\right]=0$

Updating the tableau and denoting $c_{B}^{r U} y_{b}$ by $z_{d}^{r U}$, we obtain

$x_{B}+\sum y_{d} x_{d}=\bar{b}$

$$
\begin{aligned}
& z-\sum_{r=1}^{q} w_{r}\left[\sum_{d \in D}\left\{\left(z_{d}^{r L}-c_{d}^{r L}\right)+\left(z_{d}^{r U}-c_{d}^{r U}\right)\right\} x_{d}\right] \\
& =\sum_{r=1}^{q}\left(c^{r L}(0)+\theta c^{r U}(0)\right) \bar{b}
\end{aligned}
$$

where $D$ is the set of current indices associated with the nonbasic variables $(N B)$.

For given $w^{*} \in W$, the current tableau has $\theta=\theta^{0}$ and gives an efficient basic feasible solution of $P_{0}\left(w^{*}, \theta^{0}\right)$. Our aim is to find aut haw for we can move in the direction $\theta^{r U}$ which still main taining the optimal solution of $P_{0}\left(w^{*}, \theta^{0}\right)$ problem. Let

$M=\left\{m: \sum_{r=1}^{q} w_{r}^{*}\left(z_{m}^{r U}-c_{m}^{r U} 0>0\right\}\right.$

If $M=\phi$, then the current solution is optimal to $P_{0}\left(w^{*}, \theta^{0}\right)$ problem for all values of $\theta \in\left[\theta^{0}, 1\right]$.

Otherwise, estimate the range of $\theta$ which is $\left[\theta^{0}, \theta^{1}\right]$ as:

$$
\theta^{1}=\min _{x \in M}\left\{\frac{-\sum_{r=1}^{q} w_{r}^{*}\left(z_{m}^{r L}-c_{m}^{r L}\right)}{\sum_{r=1}^{q} w_{r}^{*}\left(z_{m}^{r U}-c_{m}^{r U}\right)}\right\}
$$

Also, for determining the set of all parameters $w \in W$ corresponding to the current optimal solution of $P_{0}\left(w^{*}, \theta^{0}\right)$ problem, we calculate the ranges of $w_{r}, r=1, \ldots, q$ by using

$w_{r+1}=\min _{t \in T_{r+1}}\left\{\frac{-\left(z_{t}^{r}-c_{t}^{r}\right)}{\left(z_{r}^{r+1}-c_{r}^{r+1}\right)-\left(z_{r}^{r}-c_{r}^{r}\right)}\right\}, r=1, . ., q-1$

where

$T_{r+1}=\left\{t:\left(z_{r}^{r+1}-c_{r}^{r+1}\right) \geq 0\right.$

,and $\left.\left(z_{r}^{q}-c_{r}^{q}\right) \leq 0\right\}$

If $T_{r+1}=\phi$, then the range of $w_{r+1}$ is $\left[1-w_{r}, 1\right]$.

Otherwise, the range of $w_{r+1}$ is $\left[1-w_{r}, w_{r+1}\right]$ for all $r=1, \ldots, q-1$. 
From the above analysis, the stability set of the first kind $\left(S\left(x^{*}\right)\right)$ can be determined.

The previous procedure can be repeated until both parametric spaces of $\theta$ and $w$ are fully determined, and hence the set $E_{0}(P)$ is fully determined.

\section{NUMERICAL EXAMPLE}

To illustrate numerically Poss MOMISTP problem, we consider an example with $P=1,2=i, j$ and $k$.

(Poss MOMISTP)

$\min \tilde{z_{1}}=\sum_{p=1}^{2} \sum_{i=1}^{2} \sum_{j=1}^{2} \sum_{k=1}^{2} \tilde{c}_{i j k}^{1 p} \tilde{x}_{i j k}^{p}$,

$\min \tilde{z}_{2}=\sum_{p=1}^{2} \sum_{i=1}^{2} \sum_{j=1}^{2} \sum_{k=1}^{2} \tilde{c}_{i j k}^{2 p} \tilde{x}_{i j k}^{p}$,

subject to

$\sum_{j=1}^{2} \sum_{k=1}^{2} x_{1 j k}^{2} \leq 34, \sum_{j=1}^{2} \sum_{k=1}^{2} x_{2 j k}^{1} \leq 30$,

$\sum_{j=1}^{2} \sum_{k=1}^{2} x_{2 j k}^{2} \leq 27, \sum_{i=1}^{2} \sum_{k=1}^{2} x_{1 j k}^{1} \geq 16$,

$\sum_{i=1}^{2} \sum_{k=1}^{2} x_{1 j k}^{2} \geq 22, \sum_{i=1}^{2} \sum_{k=1}^{2} x_{i 2 k}^{1} \geq 19$,

$\sum_{i=1}^{2} \sum_{k=1}^{2} x_{i 2 k}^{2} \geq 18, \sum_{p=1}^{2} \sum_{i=1}^{2} \sum_{k=1}^{2} x_{i j 1}^{p} \leq 51$,

$\sum_{p=1}^{2} \sum_{i=1}^{2} \sum_{k=1}^{2} x_{i j 2}^{p} \leq 55$

$\left.x_{i j k}^{p} \geq 0, p=1,2 ; i=1,2 ; j=1,2 ; k=1,2\right\}$

The possibilistic variables $\quad \tilde{c}_{i j k}^{1 p}$ and $\tilde{c}_{i j k}^{2 p}$ are characterized by a possibility distributions $\left(\pi_{\tilde{c}_{i j k}^{1 p}}(\cdot)\right.$ and $\left.\pi_{\tilde{c}_{i j k}^{2 p}}(\cdot)\right)$. The supports of the possibilistic variables $\tilde{c}_{i j k}^{1 p}$ and $\tilde{c}_{i j k}^{2 p}$ are $[8,13]$ and $[9,14]$. It is appropriate to characterize the supports by a parametric functions beginning by the points of maximum possibility. Hence, the parametric function of $G$ to the supports for $\theta \in[0,1]$ are:

$\operatorname{supp} \tilde{c}_{111}^{11}=8+5 \theta, \quad \pi_{\tilde{c}_{111}^{11}}(8)=\pi_{\tilde{c}_{111}^{11}}(13)=0$ $\operatorname{supp} \tilde{c}_{111}^{12}=13-5 \theta, \pi_{\tilde{c}_{111}^{12}}(13)=\pi_{\tilde{c}_{111}^{12}}(8)=0$,

$\operatorname{supp} \tilde{c}_{211}^{11}=3+5 \theta, \quad \pi_{\tilde{c}_{211}^{11}}(3)=\pi_{\tilde{c}_{211}^{11}}(8)=0$,

$\operatorname{supp} \tilde{c}_{211}^{12}=7-5 \theta, \quad \pi_{\tilde{c}_{211}^{12}}(7)=\pi_{\tilde{c}_{211}^{12}}(2)=0$,

$\operatorname{supp} \tilde{c}_{121}^{11}=7+5 \theta, \pi_{\tilde{c}_{121}^{11}}(7)=\pi_{\tilde{c}_{121}^{11}}(12)=0$,

$\operatorname{supp} \tilde{c}_{121}^{12}=8-5 \theta, \pi_{\tilde{c}_{121}^{12}}(8)=\pi_{\tilde{c}_{121}^{12}}(3)=0$,

$\operatorname{supp} \tilde{c}_{221}^{11}=6+5 \theta, \pi_{\tilde{c}_{221}^{11}}(6)=\pi_{\tilde{c}_{221}^{11}}(11)=0$,

$\operatorname{supp} \tilde{c}_{221}^{12}=6-5 \theta, \pi_{\tilde{c}_{221}^{12}}(6)=\pi_{\tilde{c}_{221}^{12}}(1)=0$,

$\operatorname{supp} \tilde{c}_{112}^{11}=9+5 \theta, \pi_{\tilde{c}_{112}^{11}}(9)=\pi_{\tilde{c}_{112}^{11}}(14)=0$,

$\operatorname{supp} \tilde{c}_{112}^{12}=9-5 \theta, \pi_{\tilde{c}_{112}^{12}}(9)=\pi_{\tilde{c}_{112}^{12}}(4)=0$,

$\operatorname{supp} \tilde{c}_{212}^{11}=7+5 \theta, \pi_{\tilde{c}_{212}^{11}}(7)=\pi_{\tilde{c}_{212}^{11}}(12)=0$,

$\operatorname{supp} \tilde{c}_{212}^{12}=7-5 \theta, \pi_{\tilde{c}_{212}^{12}}(7)=\pi_{\tilde{c}_{212}^{12}}(2)=0$,

$\operatorname{supp} \tilde{c}_{122}^{11}=8+5 \theta, \pi_{\tilde{c}_{122}^{11}}(8)=\pi_{\tilde{c}_{122}^{11}}(13)=0$,

$\operatorname{supp} \tilde{c}_{122}^{12}=7-5 \theta, \pi_{\tilde{c}_{122}^{12}}(7)=\pi_{\tilde{c}_{122}^{12}}(2)=0$,

$\operatorname{supp} \tilde{c}_{222}^{11}=13+5 \theta, \pi_{\tilde{c}_{222}^{11}}(13)=\pi_{\tilde{c}_{222}^{11}}(18)=0$

$\operatorname{supp} \tilde{c}_{222}^{12}=13-5 \theta, \pi_{\tilde{c}_{222}^{12}}(13)=\pi_{\tilde{c}_{222}^{12}}(8)=0$,

$\operatorname{supp} \tilde{c}_{111}^{21}=9+5 \theta, \pi_{\tilde{c}_{111}^{21}}(9)=\pi_{\tilde{c}_{111}^{21}}(14)=0$,

$\operatorname{supp} \tilde{c}_{111}^{22}=9-5 \theta, \pi_{\tilde{c}_{111}^{22}}(9)=\pi_{\tilde{c}_{111}^{22}}(4)=0$,

$\operatorname{supp} \tilde{c}_{211}^{21}=2+5 \theta, \pi_{\tilde{c}_{211}^{21}}(2)=\pi_{\tilde{c}_{211}^{21}}(7)=0$,

$\operatorname{supp} \tilde{c}_{211}^{22}=8-5 \theta, \pi_{\tilde{c}_{211}^{22}}(8)=\pi_{\tilde{c}_{211}^{22}}(3)=0$,

$\operatorname{supp} \tilde{c}_{121}^{21}=15+5 \theta, \pi_{\tilde{c}_{121}^{21}}(15)=\pi_{\tilde{c}_{121}^{21}}(20)=0$,

$\operatorname{supp} \tilde{c}_{121}^{22}=15-5 \theta, \pi_{\tilde{c}_{121}^{22}}(15)=\pi_{\tilde{c}_{121}^{22}}(10)=0$,

$\operatorname{supp} \tilde{c}_{221}^{22}=13-5 \theta, \pi_{\tilde{c}_{221}^{22}}(13)=\pi_{\tilde{c}_{221}^{22}}(8)=0$, 


$$
\begin{aligned}
& \operatorname{supp} \tilde{c}_{112}^{21}=9+5 \theta, \pi_{\tilde{c}_{112}^{21}}(9)=\pi_{\tilde{c}_{112}^{21}}(14)=0, \\
& \operatorname{supp} \tilde{c}_{112}^{22}=11-5 \theta, \pi_{\tilde{c}_{112}^{22}}(11)=\pi_{\tilde{c}_{112}^{22}}(6)=0, \\
& \operatorname{supp} \tilde{c}_{212}^{21}=7+5 \theta, \pi_{\tilde{c}_{212}^{21}}(7)=\pi_{\tilde{c}_{212}^{21}}(12)=0, \\
& \operatorname{supp} \tilde{c}_{212}^{22}=6-5 \theta, \pi_{\tilde{c}_{212}^{22}}(6)=\pi_{\tilde{c}_{212}^{22}}(1)=0, \\
& \operatorname{supp} \tilde{c}_{122}^{21}=11+5 \theta, \pi_{\tilde{c}_{122}^{21}}(11)=\pi_{\tilde{c}_{122}^{21}}(16)=0, \\
& \operatorname{supp} \tilde{c}_{122}^{22}=14-5 \theta, \pi_{\tilde{c}_{122}^{22}}(14)=\pi_{\tilde{c}_{122}^{22}}(9)=0, \\
& \operatorname{supp} \tilde{c}_{122}^{21}=6+5 \theta, \pi_{\tilde{c}_{222}^{21}}(6)=\pi_{\tilde{c}_{222}^{21}}(11)=0, \\
& \operatorname{supp} \tilde{c}_{222}^{22}=7-5 \theta, \pi_{\tilde{c}_{222}^{22}}(7)=\pi_{\tilde{c}_{222}^{22}}(2)=0 .
\end{aligned}
$$

At $\alpha=0$, the corresponding 0-PMOMISTP problem is

$$
\begin{aligned}
& \min z_{1}(\theta)=(8+5 \theta) x_{111}^{1}+(13-5 \theta) x_{111}^{2}+(3+5 \theta) x_{211}^{1}+(7-5 \theta) x_{211}^{2} \\
& +(7+5 \theta) x_{121}^{1}+(8-5 \theta) x_{121}^{2}+(6+5 \theta) x_{221}^{1}+(6-5 \theta) x_{221}^{2} \\
& +(9+5 \theta) x_{112}^{1}+(9-5 \theta) x_{112}^{2}+(7+5 \theta) x_{212}^{1}+(7-5 \theta) x_{212}^{2} \\
& +(8+5 \theta) x_{122}^{1}+(7-5 \theta) x_{122}^{2}+(13+5 \theta) x_{222}^{1} \\
& +(13-5 \theta) x_{222}^{2}
\end{aligned}
$$$$
\min z_{2}(\theta)=(9+5 \theta) x_{111}^{1}+(9-5 \theta) x_{111}^{2}+(2+5 \theta) x_{211}^{1}+(8-5 \theta) x_{211}^{2}
$$$$
+(15+5 \theta) x_{121}^{1}+(15-5 \theta) x_{121}^{2}+(13+5 \theta) x_{221}^{1}+(13-5 \theta) x_{221}^{2}
$$$$
+(9+5 \theta) x_{112}^{1}+(11-5 \theta) x_{112}^{2}+(7+5 \theta) x_{212}^{1}+(6-5 \theta) x_{212}^{2}
$$$$
+(11+5 \theta) x_{122}^{1}+(14-5 \theta) x_{122}^{2}+(6+5 \theta) x_{222}^{1}
$$$$
+(7-5 \theta) x_{222}^{2} \text {, }
$$

subject to $x \in G$ and $\theta \in[0,1]$.

Also, $P_{0}(w, \theta)$ is $\min _{x \in G} w_{1} z_{1}(\theta)+w_{2} z_{2}(\theta)$

subject to

$0 \leq \theta \leq 1, w_{1}+w_{2}=1$ and $w_{1}, w_{2} \geq 0$.

Let $w^{0}=(1,0)$ and $\theta^{0}=0$. Then the corresponding $x^{0}=\left(x_{111}^{1}, x_{111}^{2}, x_{211}^{1}, x_{211}^{2}, x_{121}^{1}, x_{121}^{2}, x_{221}^{1}, x_{221}^{2}, x_{112}^{1}, x_{112}^{2}, x_{212}^{1}, x_{212}^{2}, x_{122}^{1}\right.$,

$$
\left.x_{122}^{2}, x_{222}^{1}, x_{222}^{2}\right)
$$$$
=(0,11,16,0,5,0,14,5,0,0,0,0,0,13,0,0)
$$
,and
$S\left(x^{0}\right)=\left\{\left(w^{0}, \theta^{0}\right) \in R^{3}: \frac{2}{3} \leq w_{1}^{0} \leq 1,0 \leq w_{2}^{0} \leq \frac{1}{3}\right.$, and $\left.0 \leq \theta^{0} \leq \frac{1}{8}\right\}$

Let $w^{1}=\left(\frac{2}{3}, \frac{1}{3}\right)$ and $\theta^{0}=0$. Then the corresponding efficient solution is:

$x^{0}=\left(x_{111}^{1}, x_{111}^{2}, x_{211}^{1}, x_{21}^{2}, x_{121}^{1}, x_{121}^{2}, x_{221}^{1}, x_{221}^{2}, x_{112}^{1}, x_{112}^{2}, x_{212}^{1}, x_{212}^{2}, x_{122}^{1}\right.$,

$$
\left.x_{122}^{2}, x_{222}^{1}, x_{222}^{2}\right)
$$

$=(0,11,0,0,0,0,0,0,0,0,16,0,19,0,0,18)$.

and

$S\left(x^{1}\right)=\left\{\left(w^{1}, \theta^{0}\right) \in R^{3}: \frac{1}{6} \leq w_{1}^{1} \leq \frac{4}{6}, \frac{1}{3} \leq w_{2} \frac{1}{6}, 0 \leq \theta^{0} \leq 1\right\}$

Let $w^{2}=\left(\frac{1}{6}, \frac{5}{6}\right)$ and $\theta^{0}=0$. Then the corresponding

efficient solution is:

$$
\begin{aligned}
x^{2}= & \left(x_{111}^{1}, x_{111}^{2}, x_{211}^{1}, x_{211}^{2}, x_{121}^{1}, x_{121}^{2}, x_{221}^{1}, x_{221}^{2}, x_{112}^{1}, x_{112}^{2}, x_{212}^{1}, x_{212}^{2}, x_{122}^{1},\right. \\
& \left.x_{122}^{2}, x_{222}^{1}, x_{222}^{2}\right)
\end{aligned}
$$

$=(0,11,0,0,0,0,0,0,5,0,11,0,19,0,0,18)$

and

$S\left(x^{2}\right)=\left\{\left(w^{2}, \theta^{0}\right) \in R^{3}: 0 \leq w_{1}^{2} \leq \frac{1}{6}, \frac{5}{6} \leq w_{2}^{2} \leq 1,0 \leq \theta^{0} \leq \frac{21}{32}\right\}$

Let $w^{0}=(1,0)$ and $\theta^{1}=\frac{3}{8}$. Then the corresponding

efficient solution is $x^{1}$ and

$S\left(x^{1}\right)=\left\{\left(w^{0}, \theta^{1}\right) \in R^{3}: \frac{1}{6} \leq w_{1}^{0} \leq 1,0 \leq w_{2}^{0} \leq \frac{5}{6}, \frac{3}{8} \leq \theta^{0} \leq 1\right\}$

Let $w^{1}=\left(\frac{1}{6}, \frac{5}{6}\right)$ and $\theta^{1}=\frac{3}{8}$. Then the corresponding

efficient solution is $x^{2}$ and

$S\left(x^{2}\right)=\left\{\left(w^{1}, \theta^{1}\right) \in R^{3}: 0 \leq w_{1}^{1} \leq \frac{1}{6}, \frac{5}{6} \leq w_{2}^{1} \leq 1, \frac{3}{8} \leq \theta^{1} \leq 1\right\}$

Thus,

$$
E_{0}(P)=\left\{x^{0}, x^{1}, x^{2}\right\} .
$$

\section{REFERENCES}

[1] Ammar, E. E., and Youness, E. A., (2005). Study on multi-objective transportation problem with fuzzy numbers, Applied mathematics and Computation, (166): 241-253.

[2] Ammar, E. E., and Khalifa,H.A., (2014). Study on multiobjective solid transportation problem with fuzzy numbers, European Journal of Scientific Research, (125): 7-19. 
[3] Ammar, E. E., and Khalifa,H.A., (2015). Study on possibilistic multiobjective solid transportation problems, International Journal of Current Research, (7): 1194211953.

[4] Bazaraa, M. S., Jarvis, J. J., Sherall, H. D., (1990). Linear Programming and Network Flows, Wiloy, New York, 294-299.

[5] Haley, K. B., (1962), The solid transportation problem, Operations Research, (10): 448-463.

[6] Hussein, M. L., (1998). Complete solutions of multiple objective transportation problems with possibilistic coefficients, Fuzzy Sets and Systems, (93): 293-299.

[7] Jimenez, F., Verdegay, J. L., (1998), Uncertain solid transportation problems, Fuzzy Sets and Systems, (100): 45-57.

[8] Kundu, P., Kar, S., and Maiti, M., (2013). Multiobjective multi-item solid transportation problem in fuzzy environment, Applied mathematical Modeling, (37): 2028-2038.
[9] Ojha, A., Das, B., Mondal, S., and Maiti, M., (2010). A solid transportation problem for an item with fixed charge, Vechicle cost and price discounted varying charge using genetic algorithm, Applied Soft Computing, (10): $100-110$.

[10] Luhandjula, M. K., (1987) . Multiple objective programming problems with possibilistic coefficients, Fuzzy Sets and Systems, (21): 135-145.

[11] Nanda, S. Nanda and S. Majumdar, (1992), Fuzzy Rough Sets, Fuzzy Sets and Systems, 45: 157-160.

[12] Panian, P., and Anuradha, D., (2010). A new approach for solving solid transportation problems, Applied Mathematical Sciences, (4): 3603-3610.

[13] Shell, E., (1955), Distribution of a product by several properties, Directorate of Management Analysis, Proc, $2^{\text {nd }}$ Symp. On Linear Programming (2): 615-642, DCSIComptroller H. Q. U. S. A. F., Washington, Dc.

[14] Steuer, R. E., (1986) . Multicriteria optimization: Theory, Computation and Application, Wiley, New York, 120 132. 